\title{
ADUBAÇÃO NITROGENADA EM COBERTURA PARA O FEIJOEIRO EM PLANTIO DIRETO NA PALHA ${ }^{1}$
}

\section{NITROGEN FERTILIZATION FOR DRY BEANS UNDER NO TILLAGE SYSTEM}

\author{
Sérgio Roberto PIASKOWSKI ${ }^{2}$ \\ Pedro RONZELLI JÚNIOR ${ }^{3} \equiv$ \\ Edelclaiton DAROS ${ }^{4}$ \\ Henrique Soares KOEHLER ${ }^{5}$
}

\begin{abstract}
RESUMO
Nos anos agrícolas de 1996/97 e $1997 / 98$ foram conduzidos experimentos, respectivamente, na Fazenda Escola da Universidade Estadual de Ponta Grossa e na Fazenda do Colégio Agrícola de Palmeira. Ambas as propriedades com histórico de oito anos de plantio direto na palha. O objetivo foi o de estudar a adubação nitrogenada para a cultura do feijoeiro semeado em plantio direto na palha. Avaliou-se o rendimento da cultura e seus componentes, na colheita e aspectos da morfologia e matéria seca (MS) da planta em diferentes estádios vegetativos de $V_{2}$ a $R_{9}$. O delineamento experimental foi o de blocos ao acaso, com seis tratamentos $(0,20,40,60,80$ e $100 \mathrm{~kg}$ de N.ha ${ }^{-1}$ ) e cinco repetições. Utilizou-se como indicadora a variedade de feijão 'FT Nobre'. Mesmo tendo sido feitas muitas determinações apenas algumas mostraram efeito dos tratamentos que fossem estatisticamente significativos. $O$ rendimento de grãos e seus componentes comprovaram, em plantio direto na palha, a instabilidade do efeito do nitrogênio para a cultura do feijoeiro, de qualquer modo, independente de diferenças estatisticamente significativas, em ambos os experimentos foram obtidas produtividades bastante altas que, em média, superam as produtividades do Estado do Paraná em mais de duas vezes e a brasileira em mais de quatro vezes. Nos dois experimentos, tanto os aspectos morfológicos quanto a determinação da MS da planta ou de parte dela mostraram resultados concordantes com os do rendimento de grãos. Assim, concluiu-se que não é possível assegurar que a presença de matéria orgânica em maior abundância, comum no plantio direto na palha, resulte em menor exigência da cultura do feijoeiro por nitrogênio e que se confirma, também para plantio direto na palha, que o nitrogênio é nutriente de respostas variadas, nem sempre de acordo com o esperado para a cultura do feijoeiro.

Palavras-chave: Phaseolus vulgaris, nitrogênio, plantio direto.
\end{abstract}

\begin{abstract}
Field trials were carried out in the agricultural years of 1996/97 and 1997/98, respectively, at the Universidade Estadual de Ponta Grossa Farm School, and at the Colégio Agrícola de Palmeira Farm. Both places have eight years history of no tillage system. The objective was to study nitrogen fertilization for dry beans under no till system. There were evaluated yield and their components of the dry beans culture at harvest, and morphological modifications and dry matter of the plant at different vegetative stadium, from $V_{2}$ to $R_{9}$. The experimental design employed was a randomized complete block, with six treatments $\left(0,20,40,60,80\right.$ and $100 \mathrm{~kg}$ of N.ha $\left.{ }^{-1}\right)$ and five replications with the 'FT Nobre' dry beans variety. Even considering the large number of variables determination, only a few showed significant statistical differences. The yield and components confirmed the nitrogen effect instability on no till system for dry beans culture. Anyway, disconsidering the statistical differences, both experiments showed high yield, like more then twice the State of Paraná average and like four times the country one. Both experiments showed that morphological aspects and MS determination of the plant or parts of them, produced results that agree with the yield results. The conclusion is that it is not possible to confirm that the organic matter presence, very common on no till system, conduct to less exigency for nitrogen and confirm that the dry beans culture answers for nitrogen fertilization, either at no till system, are so variable.

Key words: Phaseolus vulgaris, nitrogen, no till system.
\end{abstract}

\footnotetext{
${ }^{1}$ Parte da dissertação do primeiro autor, apresentada na Universidade Federal do Paraná, para obter o grau de Mestre em Ciências no Curso de Pós Graduação em Agronomia, Produção Vegetal.

2 Engenheiro Agrônomo, M.Sc., Companhia Nacional de Abastecimento - CONAB

${ }^{3}$ Engenheiro Agrônomo, Doutor, Universidade Federal do Paraná, Professor Adjunto IV, Bolsista do CNPq. Email: agroprj@.ufpr.br -- Autor para correspondência.

${ }^{4}$ Engenheiro Agrônomo, Doutor, Universidade Federal do Paraná, Professor Adjunto IV.

${ }^{5}$ Engenheiro Florestal, Doutor, Universidade Federal do Paraná, Professor Adjunto IV.
} 


\section{INTRODUÇÃO}

O feijão é um dos alimentos básicos da dieta do brasileiro, muito importante por ser fonte de proteína. O Paraná um dos principais produtores, onde a cultura do feijoeiro é tradicional entre mini e pequenos produtores. No entanto, nos últimos anos, tem se observado tendência de alteração desse perfil $[3,11,16]$.

O nitrogênio, nutriente fundamental para a cultura do feijoeiro é elemento de alta mobilidade no sistema solo-planta-atmosfera, perdendo-se facilmente por volatilização ou lixiviação e, além disso, os adubos nitrogenados têm baixa eficiência e alto custo de sintetização, o que permite considerar que sua utilização sem critério, além de elevar o custo do produto final, pode contaminar o ambiente [3,12].

O sistema de plantio direto na palha faz parte das tecnologias tidas como modernas. Para outras espécies vegetais já tem sido utilizado com vantagens e constata-se, ano após ano, aumento nas áreas em cultivo nesse sistema. Tem se observado, também, que tem havido interesse em adotar a tecnologia, inclusive pelos mini e pequenos agricultores [2, 11, 16].

Em condições de plantio direto na palha, em razão da presença mais intensa de matéria orgânica no solo, a cultura do feijoeiro deve ser menos exigente em adubação nitrogenada de cobertura. Assim avaliou-se o rendimento e seus componentes, modificações morfológicas e matéria seca da planta. As informações obtidas são úteis tanto para os que já utilizam o sistema de semeadura direta em outras culturas, quanto para a difusão dessa técnica, ainda incomum para a cultura do feijoeiro.

\section{METODOLOGIA}

O trabalho experimental foi desenvolvido a campo em dois anos agrícolas. No primeiro (1996/97), na Fazenda Experimental da Universidade Estadual de Ponta Grossa (UEPG), em Ponta Grossa, PR e no segundo (1997/98), na Fazenda do Colégio Agrícola de Palmeira (CAP), em Palmeira, PR. Ambos os locais têm clima Cfb, segundo Köepen [7].

$O$ delineamento experimental foi o de blocos ao acaso com seis tratamentos $0,20,40,60,80$ e $100 \mathrm{~kg}$ de N.ha ${ }^{-1}$, e cinco repetições. O nitrogênio, tendo como fonte a uréia, foi colocado a lanço, no estádio $V_{3}(50 \%$ das plantas com a primeira folha trifoliolada completamente aberta) [4]. Foi utilizada a variedade de feijão 'FT-Nobre'. As variâncias dos tratamentos foram testadas quanto a homogeneidade pelo teste de Bartlett. Quando homogêneas os efeitos dos tratamentos foram testados pelo teste de $\mathrm{F}$ a $5 \mathrm{e}$ $1 \%$ de probabilidade. As variáveis cujas médias mostraram diferenças significativas foram comparadas pelo teste de Tukey a $5 \%$ de probabilidade.

\section{Experimento 1}

O solo da área utilizada é um Latossolo Vermelho Escuro Álico, A-proeminente, textura argilosa, fase campo subtropical, relevo suave [13]. Foram coletadas amostras na faixa de 0 a $20 \mathrm{~cm}$ para análises química e física. Os resultados dessas análises são apresentados no Quadro 1.

QUADRO 1 - Características químicas e percentual de argila do solo da área experimental, camada de 0 a $20 \mathrm{~cm}$, Fazenda Experimental da Universidade Estadual de Ponta Grossa, Ponta Grossa, PR, $1996 / 1997^{1}$

\begin{tabular}{|c|c|c|c|c|c|c|c|c|c|c|c|}
\hline $\mathrm{pH}$ & $\mathrm{Al}^{+3}$ & $\mathrm{H}+\mathrm{Al}^{+3}$ & $\mathrm{Ca}+\mathrm{Mg}^{+2}$ & $\mathrm{~K}^{+1}$ & $\mathrm{~S}^{+3}$ & $\mathrm{~T}$ & $P$ & $\mathrm{C}$ & $m$ & $\mathrm{~V}$ & Argila \\
\hline $\mathrm{CaCl}_{3}$ & \multicolumn{6}{|c|}{$\mathrm{cmol}_{\mathrm{c} .} \mathrm{dm}^{-3}$} & $\mathrm{mg} \cdot \mathrm{dm}^{-3}$ & g.dm ${ }^{-3}$ & & $\%$ & \\
\hline 5,0 & 0,0 & 6,2 & 4,70 & 0,37 & 5,99 & 11,27 & 1,00 & 25,9 & 00,0 & 45,0 & 56,0 \\
\hline
\end{tabular}

A unidade experimental era composta por oito fileiras com espaçamentos, entre si, de $0,45 \mathrm{~m}$, perfazendo área total de $21,60 \mathrm{~m}^{2}$. Das oito fileiras quatro foram utilizadas para colher as dez plantas necessárias para as avaliações de matéria seca (MS) e características morfológicas das plantas. Das quatro fileiras restantes as duas laterais foram consideradas como bordaduras e nas duas centrais, desconsiderados os $0,50 \mathrm{~m}$ de cada extremidade, perfazendo uma área útil de $4,50 \mathrm{~m}^{2}$, foram colhidas as plantas para a avaliação do rendimento, separando-se dez para a avaliação dos componentes do rendimento.

A área experimental, antes da semeadura do feijão, estava coberta com a cultura do trigo em fase vegetativa. Essa vegetação foi dessecada, em 26 SET 1996, utilizando-se glifosate, na dose de
2,0 L.ha' ${ }^{-1}$. Em seguida foi feito o manejo mecânico da cultura dessecada com um "rolo-faca". Em 30 SET 1996 foi feita a semeadura do feijão. Foram colocadas 15 sementes por metro, visando uma população de trezentas mil plantas por hectare. No sulco de semeadura foi colocada a adubação de base com $360 \mathrm{~kg} \cdot \mathrm{ha}^{-1}$ do formulado 2-25-25. A emergência foi identificada em 07 OUT 1996, observando-se desuniformidade.

Os tratos fitossanitários foram feitos com duas aplicações seqüenciais da mistura comercial dos herbicidas fluazifop-p-butil + fomesafem, na dose de 0,5 L.ha $^{-1}$, cada uma, nos estádios $V_{3}$ e $V_{4}$, para $o$ controle de plantas daninhas, e uma aplicação dos fungicidas tiofanato metilico + clorothalonil, na dose de $2,5 \mathrm{~kg} \cdot \mathrm{ha}^{-1}$, para o controle das principais doenças fúngicas das folhas e uma aplicação do inseticida 
methamidophos na proporção de 1,0 L.ha ${ }^{-1}$, para o controle das principais pragas da cultura, essas duas últimas no estádio $\mathrm{V}_{4}$.

As avaliações de matéria seca (MS) foram feitas nos estádios de desenvolvimento $V_{2}, V_{3}, V_{4}$, $R_{5}, R_{6}, R_{7}$ e $R_{9}$. Para isso as plantas foram colhidas, secas em estufa a $70^{\circ} \mathrm{C}$, até atingir massa constante e, então, determinada a MS. $E m \mathrm{R}_{9}$ os valores de MS foram separados em MS total, das vagens e da semente, em relação ao caule, aos ramos e total. Em $\mathrm{R}_{9}$ foram também determinados a estatura, o número total de ramos, o número total de vagens no caule, nos ramos e na planta e o número total de sementes no caule, nos ramos e na planta. $\mathrm{Na}$ área útil, na colheita $\left(\mathrm{R}_{9}\right)$, em dez plantas foram avaliados os números totais de vagens e sementes. Por meio desses valores foram calculados os componentes do rendimento, número médio de vagens por planta (NMVP) e número médio de sementes por vagem (NMSV). A massa média de 100 grãos (MM100G), foi determinada a partir de três amostras de 100 grãos de cada parcela, que depois foram corrigidas para $13 \%$ de umidade relativa. O rendimento foi obtido das plantas da área útil, inclusive as dez utilizadas para avaliação dos componentes do rendimento, sendo os valores corrigidos para $13 \%$ de umidade relativa.

\section{Experimento 2}

O solo da área utilizada é um Cambissolo [13]. Foram coletadas amostras na faixa de 0 a $20 \mathrm{~cm}$ para análises química e física. Os resultados dessas análises são apresentados no Quadro 2.

QUADRO 2 - Características químicas e percentual de argila do solo da área experimental, camada de 0 a $20 \mathrm{~cm}$, Colégio Agrícola de Palmeira, PR, 1997/98 ${ }^{1}$

\begin{tabular}{|c|c|c|c|c|c|c|c|c|c|c|c|}
\hline $\mathrm{pH}$ & $\mathrm{Al}^{+3}$ & $\mathrm{H}+\mathrm{Al}^{+3}$ & $\mathrm{Ca}+\mathrm{Mg}^{+2}$ & $\mathrm{~K}^{+1}$ & $\mathrm{~S}^{+3}$ & $\mathrm{~T}$ & P & C & $\mathrm{m}$ & V & Argila \\
\hline $\mathrm{CaCl}_{3}$ & \multicolumn{6}{|c|}{$\mathrm{cmol}_{\mathrm{c}} \cdot \mathrm{dm}^{-3}$} & $\mathrm{mg} \cdot \mathrm{dm}^{-3}$ & g.dm ${ }^{-3}$ & & $\%$ & \\
\hline 5,1 & 0,07 & 4,80 & 5,86 & 0,12 & 5,99 & 10,79 & 4,60 & 13,3 & 1,16 & 55,7 & 32,0 \\
\hline
\end{tabular}

A unidade experimental era composta por oito fileiras com espaçamentos, entre si, de $0,40 \mathrm{~m}$ perfazendo área total de $20,80 \mathrm{~m}^{2}$. Das oito fileiras quatro foram utilizadas para colher as dez plantas necessárias para as avaliações de MS e características morfológicas das plantas. Das quatro restantes, as duas laterais foram consideradas como bordaduras e nas duas centrais, desconsiderados os $0,25 \mathrm{~m}$ de cada extremidade, foram colhidas as plantas para a avaliação do rendimento, numa área útil de $4,80 \mathrm{~m}^{2}$.

A área experimental estava coberta com a cultura da aveia preta em fase vegetativa. Essa vegetação foi dessecada em 26 NOV 1997, utilizandose sulfosate, na dose de 2,0 L.ha ${ }^{-1}$. Em 05 DEZ 1997 foi feita a semeadura do feijão, colocando-se 16 sementes por metro, visando uma população de quatrocentas mil plantas por hectare. No sulco de semeadura foi colocada adubação de base com $300 \mathrm{~kg} \cdot \mathrm{ha}^{-1}$ do formulado 0-30-10. A emergência foi identificada como uniforme em 10 DEZ 1997.

Os tratos fitossanitários foram feitos com uma aplicação da mistura comercial dos herbicidas fluazifop-p-butil + fomesafem, na dose de 1,0 L.ha ${ }^{1}$, para controle das plantas daninhas, no estádio $V_{3}$, e uma aplicação dos fungicidas tiofanato metílico + clorothalonil na dose de $2,5 \mathrm{~kg} \cdot \mathrm{ha}^{-1}$, para o controle das principais doenças fúngicas das folhas e uma aplicação do inseticida methamidophos na dose de 1,0 L.ha ${ }^{-1}$, para o controle das principais pragas da cultura, essas duas últimas no estádio $\mathrm{V}_{4}$.

As avaliações de MS foram feitas nos estádios de desenvolvimento $V_{2}$, a $R_{9}$. Para isso as plantas foram colhidas, secas em estufa a $70^{\circ} \mathrm{C}$, até atingir massa constante e, então, determinada a MS. Em $V_{2}$ e $V_{3}$ os valores de MS foram separados em
MS total, do caule, dos pecíolos e das folhas. Em $V_{4}$ os valores de MS foram separados em MS total, do caule, dos ramos, dos pecíolos e das folhas. Em $\mathrm{R}_{5}$ os valores de MS foram separados em MS total, do caule, dos ramos, dos pecíolos, das folhas e das flores. $E m R_{6} \quad a R_{8}$ os valores de $M S$ foram separados em MS total, de caule, ramos e pecíolos, MS total das vagens de caule e ramos e MS total das sementes de caule e ramos.

A estatura de caule e de ramos foi determinada de $R_{5}$ a $R_{9}$. Na colheita $\left(R_{9}\right)$, em dez plantas da área útil foram determinados os números totais de vagens e sementes. Por meio desses valores foram calculados os componentes do rendimento: número médio de vagens por planta (NMVP) e número médio de sementes por vagem (NMSV). A massa média de 100 grãos (MM100G), foi determinada a partir de três amostras de 100 grãos de cada parcela, que depois foram corrigidas para $13 \%$ de umidade relativa. 0 rendimento foi obtido das plantas da área útil, inclusive as dez utilizadas para avaliação dos componentes do rendimento, sendo os valores corrigidos para $13 \%$ de umidade relativa.

\section{RESULTADOS E DISCUSSÃO}

Em ambos os experimentos, mesmo tendo sido feitas muitas determinações, foram poucas as que mostraram efeito estatisticamente significativo dos tratamentos. O rendimento de grãos e os componentes do rendimento são bons exemplos para demonstrar essa instabilidade do efeito do nitrogênio em cobertura para a cultura do feijoeiro, conforme já havia sido demonstrado por Malavolta, 
1971 [10]. Na Tabela 1 são apresentados os resultados de rendimento de grãos dos dois experimentos.

TABELA 1 - Rendimento da cultura do feijoeiro, variedade 'FT Nobre', nos Experimento 1 (Fazenda Escola da Universidade Estadual de Ponta Grossa), Ponta Grossa, PR, 1996/97 e Experimento 2 (Colégio Agrícola de Palmeira), Palmeira, PR, 1997/98 ${ }^{1}$

\begin{tabular}{ccc}
\hline $\begin{array}{c}\text { Tratamento } \\
\left(\text { kg N.ha }^{-1}\right)\end{array}$ & \multicolumn{2}{c}{ Rendimento (kg.ha $\left.{ }^{-1}\right)$} \\
\cline { 2 - 3 } & Experimento 1 & Experimento 2 \\
\hline 0 & 1.614 & $1.976 \mathrm{ab}$ \\
20 & 1.730 & $1.805 \mathrm{~b}$ \\
40 & 1.638 & $2.236 \mathrm{a}$ \\
60 & 2.144 & $2.092 \mathrm{ab}$ \\
80 & 2.124 & $2.200 \mathrm{a}$ \\
100 & 2.207 & $2.095 \mathrm{ab}$ \\
\hline $\mathrm{CV}(\%)^{2}$ & - & 9,30 \\
\hline${ }^{2}$ Médias seguidas da mesma letra não diferem \\
significativamente pelo teste de Tukey a 5\% de probabilidade. \\
${ }^{2} \mathrm{CV}=$ Coeficiente de Variação
\end{tabular}

Observa-se pela Tabela 1 que foram identificadas diferenças estatisticamente significativas apenas no Experimento 2. Independentemente da informação estatística podese ver que os rendimentos foram muito bons superando médias do Paraná (832 kg.ha $\left.{ }^{-1}\right)$ e brasileira (592 kg.ha-1 $)$ [15]. No Experimento 1 ocorreu o esperado, ou seja, foram observados maiores rendimentos nas doses maiores de nitrogênio em cobertura. Apesar de não terem sido identificadas diferenças estatísticas significativas houve variação de $593 \mathrm{~kg} \cdot \mathrm{ha}^{-1}$ entre o melhor e o pior resultado de rendimento. No Experimento 2, considerando o resultado estatístico significativo, não foram identificadas diferenças entre os tratamentos com adubação em cobertura, desde $40 \mathrm{~kg}$ de N.ha ${ }^{-1}$ até $100 \mathrm{~kg}$ de N.ha ${ }^{-1}$. Nota-se, semelhantemente ao experimento anterior, que existe uma diferença grande entre o melhor e o pior resultado de rendimento de grãos, neste caso de $431 \mathrm{~kg}$.ha-1. Essas diferenças entre melhor e pior resultados equivalentes a aproximadamente 11 sacas de $50 \mathrm{~kg}$ para o Experimento 1 e nove para - Experimento 2 não podem ser desprezadas comercialmente, uma vez que são valores muito próximos da média brasileira de produtividade.

\section{Experimento 1}

$\mathrm{Na}$ Tabela 2 são apresentados os resultados com diferenças estatisticamente significativas de características morfológicas e MS. Pode-se ver que as características morfológicas somente mostraram essas diferenças no estádio $R_{9}$ e que para $M S$ ocorreram nos estádios $R_{6}$ e $R_{9}$. Para MS, observase no estádio $R_{6}$, que apenas o tratamento sem nitrogênio foi estatisticamente inferior aos demais. No estádio $R_{9}$, nota-se que houve, tanto para a MS do caule quanto para a total, semelhança ao descrito em $R_{6}$, destacando-se, neste caso, um tratamento superior estatisticamente que foi o com a maior dose de N. Pode-se ver, também, que as características morfológicas avaliadas acompanharam a tendência observada para MS, destacando-se como superiores estatisticamente os tratamentos com maiores doses de $\mathrm{N}$. No entanto, como já discutido anteriormente, essas diferenças mesmo tendo sido identificadas no rendimento, não foram significativas para aquela variável. Resta considerar que a análise estatística, às vezes, precisa ser tratada com muito cuidado, já que os valores reais parecem oferecer mais segurança quando se associam os resultados obtidos de rendimento e características da cultura e da planta.

TABELA 2 - Características morfológicas e matéria seca da cultura do feijoeiro, variedade 'FT Nobre' submetida a seis doses de nitrogênio em plantio direto na palha, Fazenda Escola da Universidade Estadual de Ponta Grossa, Ponta Grossa, PR, 1996/97 ${ }^{1}$

\begin{tabular}{|c|c|c|c|c|c|c|c|c|}
\hline \multirow{4}{*}{$\begin{array}{l}\text { Tratamento } \\
\left(\mathrm{kg} \mathrm{N} \mathrm{ha}^{-1}\right)\end{array}$} & \multicolumn{8}{|c|}{ Estádios } \\
\hline & \multirow{3}{*}{$\begin{array}{c}\mathrm{R}_{6} \\
\text { Matéria } \\
\text { Seca Total } \\
\text { (g) }\end{array}$} & \multicolumn{7}{|c|}{$\mathrm{R}_{9}$} \\
\hline & & \multicolumn{2}{|c|}{$\begin{array}{c}\text { Matéria Seca } \\
(\mathrm{g})\end{array}$} & \multirow{2}{*}{$\begin{array}{c}\text { Número } \\
\text { de } \\
\text { Ramos } \\
\text { Total }\end{array}$} & \multicolumn{2}{|c|}{ Número de Vagens } & \multicolumn{2}{|c|}{$\begin{array}{l}\text { Número de } \\
\text { Sementes }\end{array}$} \\
\hline & & Caule & Total & & Caule & Total & Caule & Total \\
\hline 0 & $28,56 \mathrm{~b}$ & $1.658 \mathrm{bc}$ & $3.314 \mathrm{c}$ & $3,28 a b$ & $6,1 \mathrm{~b}$ & $17,0 \mathrm{~b}$ & $30,2 \mathrm{bc}$ & $71,6 \mathrm{~b}$ \\
\hline 20 & $41,42 a b$ & $1.526 \mathrm{c}$ & $3.556 \mathrm{bc}$ & $3,12 b$ & $6,0 \mathrm{~b}$ & $17,9 a b$ & $28,6 \mathrm{c}$ & $81,7 a b$ \\
\hline 40 & $43,36 a b$ & $1.770 \mathrm{bc}$ & $4.376 \mathrm{abc}$ & $3,82 a b$ & $7,0 a b$ & $22,0 a b$ & $33,0 \mathrm{abc}$ & $92,2 a b$ \\
\hline 60 & 58,18 a & $1.942 a b c$ & $4.156 a b c$ & $3,62 a b$ & $7,4 a b$ & $20,6 a b$ & $37,1 \mathrm{abc}$ & $89,9 a b$ \\
\hline 80 & $58,70 \mathrm{a}$ & $2.154 a b$ & $5.014 a b$ & $4,06 \mathrm{a}$ & $9,0 \mathrm{a}$ & $25,3 \mathrm{a}$ & $41,0 a b$ & $114,4 \mathrm{a}$ \\
\hline 100 & $42,66 a b$ & $2.380 \mathrm{a}$ & $5.260 \mathrm{a}$ & $4,04 \mathrm{a}$ & $8,4 \mathrm{a}$ & $25,0 \mathrm{a}$ & $42,1 \mathrm{a}$ & $107,2 \mathrm{a}$ \\
\hline CV $(\%)^{2}$ & 23,22 & 14,90 & 17,70 & 11,40 & 14,50 & 17,70 & 16,70 & 21,70 \\
\hline
\end{tabular}

1 Médias seguidas pela mesma letra não diferem estatisticamente pelo teste de Tukey a $5 \%$ de probabilidade.

$2 \mathrm{CV}=$ Coeficiente de Variação em percentagem. 
Comparando-se estes resultados com os obtidos em sistema de semeadura convencional, observa-se que podem ser relacionados como concordantes e discordantes em relação aos diversos autores que trabalharam com a cultura do feijoeiro $[1,5,6,8,9,10,15]$. Provavelmente, a melhor interpretação para a variação nos resultados é dada por Malavolta, 1971 [10], que para adubação nitrogenada na cultura do feijoeiro em sistema convencional de preparo de solo, concluiu que apenas $30 \%$ dos trabalhos experimentais mostraram resultados da eficiência do nitrogênio para a cultura.

Considera-se, também, que a população inicial desuniforme foi determinante nos resultados apresentados. Uma possível interação alelopática da palha do trigo, que se manifestou da emergência até a colheita, é reforçada pelo fato de, em outro experimento conduzido em área contígua e com outro objetivo, onde não foi realizado o manejo mecânico da cobertura com o equipamento "RoloFaca" não ter sido observada desuniformidade na emergência e estabelecimento da cultura. Almeida, 1988 [2] relata interferência semelhante.

\section{Experimento 2}

Na Tabela 3 são apresentados os resultados com diferenças estatisticamente significativas de características morfológicas e MS. Observam-se essas diferenças para características morfológicas nos estádios $R_{5}, R_{7}, R_{8}$ e $R_{9}$ e para MS no estádio $\mathrm{R}_{8}$.

TABELA 3 - Características morfológicas e matéria seca da cultura do feijoeiro, variedade 'FT Nobre', submetida a seis doses de nitrogênio em plantio direto na palha, Colégio Agrícola de Palmeira, Palmeira, PR, 1997/98 ${ }^{1}$

\begin{tabular}{|c|c|c|c|c|c|c|c|}
\hline \multirow{4}{*}{$\begin{array}{l}\text { Tratamento } \\
\left(\mathrm{kg} \mathrm{N}^{-h^{-1}}\right)^{-}\end{array}$} & \multicolumn{7}{|c|}{ Estádios } \\
\hline & \multirow{3}{*}{$\begin{array}{c}\mathrm{R}_{5} \\
\text { Estatura do } \\
\text { Caule } \\
\text { (cm) }\end{array}$} & \multicolumn{2}{|c|}{$\mathrm{R}_{7}$} & \multirow{3}{*}{$\begin{array}{c}\mathrm{R}_{8} \\
\text { Estatura do } \\
\text { Caule } \\
(\mathrm{cm})\end{array}$} & \multirow{2}{*}{\multicolumn{3}{|c|}{$\begin{array}{c}\mathrm{R}_{9} \\
\text { Matéria Seca } \\
(\mathrm{g})\end{array}$}} \\
\hline & & Estatura do & $\begin{array}{l}\text { Matéria } \\
\text { Seca do }\end{array}$ & & & & \\
\hline & & (cm) & $\begin{array}{c}\text { Caule } \\
\text { (g) }\end{array}$ & & Ramos & $\begin{array}{c}\text { Sementes do } \\
\text { Caule }\end{array}$ & $\begin{array}{l}\text { Sementes } \\
\text { Totais }\end{array}$ \\
\hline 0 & $28,6 a b$ & $59,06 \mathrm{c}$ & $1,92 b$ & $61,32 b$ & $0,29 \mathrm{~b}$ & $6,92 \mathrm{~b}$ & $10,30 \mathrm{~b}$ \\
\hline 20 & $28,0 a b$ & 69,42 bc & $2,02 a b$ & $64,16 \mathrm{~b}$ & $0,41 b$ & $7,29 a b$ & $11,09 a b$ \\
\hline 40 & $32,5 \mathrm{a}$ & 70,14 abc & $2,26 a b$ & $66,96 \mathrm{~b}$ & $0,43 b$ & $8,49 a b$ & $12,33 a b$ \\
\hline 60 & $31,3 a b$ & $79,72 \mathrm{ab}$ & $2,38 \mathrm{ab}$ & $74,36 \mathrm{~b}$ & $0,58 \mathrm{~b}$ & $8,69 a$ & $13,24 a$ \\
\hline 80 & $26,9 \mathrm{~b}$ & 82,06 a & $2,48 a$ & $73,64 a b$ & $0,40 \mathrm{~b}$ & $7,72 \mathrm{ab}$ & $11,61 a b$ \\
\hline 100 & $27,3 \mathrm{~b}$ & 69,66 abc & $2,34 \mathrm{ab}$ & 84,56 a & $0,89 a$ & $7,82 \mathrm{ab}$ & $12,17 a b$ \\
\hline CV (\%) & 8,60 & 11,40 & 11,80 & 12,20 & 24,50 & 11,10 & 11,30 \\
\hline
\end{tabular}

Vê-se, pela Tabela 3, que a MS nos estádio $R_{7}$, apenas do caule, e $R_{9}$, de ramos, sementes do caule e totais, à semelhança do que foi observado no Experimento 1, tem apenas o tratamento sem nitrogênio como estatisticamente inferior aos demais. Vê-se, também, tendência de às maiores doses de nitrogênio estarem associados maiores valores de MS. Esses resultados confirmam o resultado de rendimento obtido.

Quanto à estatura do caule, vê-se, também pela Tabela 3, que apenas no estádio $R_{5}$ houve resposta estatisticamente diferenciada para tratamentos com dose menor de $\mathrm{N}$. Nos estádios $\mathrm{R}_{7}$ e $R_{8}$ confirma-se a tendência de melhores resultados com maiores doses.

\section{CONCLUSÕES}

1) Não é possível assegurar que a presença de matéria orgânica em maior abundância, comum no plantio direto na palha, resulte em menor exigência da cultura do feijoeiro por nitrogênio.

2) Confirma-se, para plantio direto na palha, que o nitrogênio é nutriente de respostas variadas, nem sempre de acordo com o esperado para a cultura do feijoeiro.

\section{REFERÊNCIAS}

[1] ALMEIDA, A. A.; LOPES, N. F.; OLIVA, M. A.; BARROS, R. S. Doses de nitrogênio e densidades de luz no crescimento do feijoeiro e na conversão da energia solar. Pesquisa Agropecuária Brasileira, Brasília, v.23, n.8, p. 849-859, 1988.

[2] ALMEIDA, F. S. A alelopatia e as plantas. Londrina: IAPAR, 60 p. 1988. (Circular 53)

[3] BALOTA, E. L. Alterações microbiológicas em solo cultivado sob o plantio direto. In: PEIXOTO, R. T. G.; AHRENS, D. C.; SAMAHA, M. J. Plantio direto: o caminho para uma agricultura sustentável. Ponta Grossa: IAPAR, 1997. p. 222-231.

[4] FERNANDES, F.; GEPTS, P.; LOPEZ, M. Etapas de desarrollo de la planta de frijol común. Cali: CIAT, 1982. $26 \mathrm{p}$.

[5] FORNASIERI FILHO, D.; BELLINDIERI, P. A.; VITTI, G. C.; MALHEIROS, E. B.; HORIENTE, E. C. Efeitos da inoculação com Rhizobium phaseoli de fertilizantes as sementes e nitrogênio mineral na cultura do feijoeiro (Phaseolus vulgaris L.) Carioca das "águas". Científica, São Paulo, v.16, n.2, p. 229-238, 1988. 
[6] FORNASIERI FILHO, D.; VITTI, G. C.; MALHEIROS, E. B.; DECARO, S.; LÁM-SANCHES, A. Efeito da inoculação associada a aplicação de micronutrientes e nitrogênio mineral na cultura do feijoeiro cv. Carioca 80. Científica, São Paulo, v.16, n.2, p. 197-207, 1988.

[7] GODOI, H.; CORRÊA, A. R. Cartas Climáticas básicas do Estado do Paraná. Londrina: Fundação Instituto Agronômico do Paraná, 1978. 38 p.

[8] MACHADO, J. R.; ROSOLEM, C. A.; NAKAGAWA, J. N. Adubação foliar e épocas de aplicação do adubo nitrogenado. In: REUNIÂO NACIONAL DE PESQUISA DE FEIJÃO, 1., Goiânia, 1982. Anais. Goiânia: EMBRAPA, 1982. p. 196-198.

[9] MAFRA, R. C.; PORTELA, M. C. L. S.; PEREIRA, J. T. Manejo da adubação nitrogenada, fontes, épocas de aplicação e sistema de parcelamento na cultura do feijão (Phaseolus vulgaris L.). In: REUNIÃO NACIONAL DE PESQUISA DE FEIJÃO, 1., Goiânia, 1982. Anais. Goiânia: EMBRAPA, 1982. p. 177-178.

[10] MALAVOLTA, E. Nutrição e Adubação. In SIMPÓSIO BRASILEIRO DE FEIJÃO, 1., Campinas, 1971. Anais. Viçosa: UFV - Imprensa Universitária, 1972. p. 211-242.

[11]MALAVOLTA, E.; VITTI, G. C.; OLIVEIRA, S. A. Avaliação do estado nutricional das plantas: princípios e aplicações. Piracicaba: Associação Brasileira para Pesquisa da Potassa e do Fosfato, 1989. $201 \mathrm{p}$
[12]MORAES, J. F V Calagem e adubação In: ZIMMERMANN, M. J. O.; ROCHA. M.; YAMADA, T. Cultura do feijoeiro: fatores que afetam a produtividade. Piracicaba: Associação Brasileira para Pesquisa da Potassa e do Fosfato, 1988. p. 261-301.

[13]OLMOS-ITURRI, L. J.; CARDOSO, A.; CARVALHO, A. P.; HOCHMÜLER, D. P.; FASOLO, P. J.; RAÜEN, M. J. Levantamento de reconhecimento dos solos do Estado do Paraná. Londrina: EMBRAPA/SNLCS/SUDESUL/IAPAR, 1984.412 p. (Boletim Técnico 57)

[14]ROMEIRO, A. D. Agricultura sustentável e tecnologia. In: PEIXOTO, R .T. G.; AHRENS, D. C.: SAMAHA, M. J. Plantio direto: o caminho para uma agricultura sustentável. Ponta Grossa: IAPAR, 1997. p. 2-8.

[15]VIEIRA, C.; GOMES, F. R. Ensaios de adubação química do feijoeiro. Revista Ceres, Viçosa, v.11, n.65, p. 253-264, 1961.

[16]YOKOYAMA, L. P.; BANNO, K.; KLUTHCOUSKI, J. Aspectos socioeconômicos da cultura. In: ARAUJO, R. S.; RAVA. C. A.; STONE, L. F.; ZIMMERMANN, M. J. O. Cultura do feijoeiro comum no Brasil. Piracicaba: Associação Brasileira para Pesquisa da Potassa e do Fosfato, 1996. p. 1-21.

Recebido para publicação em 16 AGO 2000 [SA 026/2000] Aceito para publicação em 12 SET 2001 